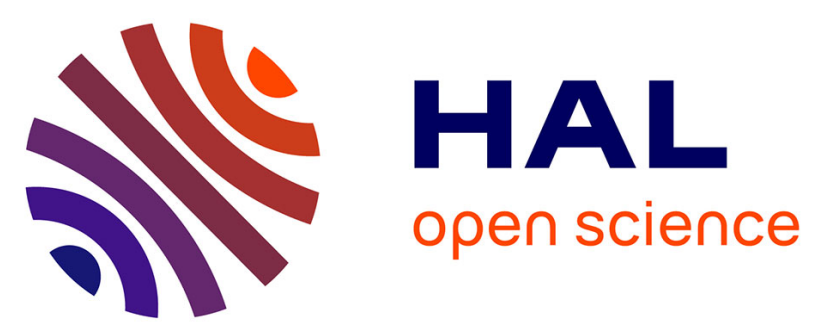

\title{
Présentation d'un support informatique de reconnaissance de mots écrits pour des enfants non verbaux avec autisme
}

Frédérique Vernay, Jean-Yves Roussey

\section{- To cite this version:}

Frédérique Vernay, Jean-Yves Roussey. Présentation d'un support informatique de reconnaissance de mots écrits pour des enfants non verbaux avec autisme. Enfance, 2012, 64 (4), pp.411 - 422. 10.4074/S0013754512004041 . hal-01709922

\section{HAL Id: hal-01709922 \\ https://hal-amu.archives-ouvertes.fr/hal-01709922}

Submitted on 15 Feb 2018

HAL is a multi-disciplinary open access archive for the deposit and dissemination of scientific research documents, whether they are published or not. The documents may come from teaching and research institutions in France or abroad, or from public or private research centers.
L'archive ouverte pluridisciplinaire $\mathbf{H A L}$, est destinée au dépôt et à la diffusion de documents scientifiques de niveau recherche, publiés ou non, émanant des établissements d'enseignement et de recherche français ou étrangers, des laboratoires publics ou privés. 


\section{PRÉSENTATION D’UN SUPPORT INFORMATIQUE DE RECONNAISSANCE DE MOTS ÉCRITS POUR DES ENFANTS NON VERBAUX AVEC AUTISME \\ Frédérique Vernay, Jean-Yves Roussey}

NecPlus | «nfance »

2012/4 N 4 | pages 411 à 422

ISSN 0013-7545

Article disponible en ligne à l'adresse :

https://www.cairn.info/revue-enfance2-2012-4-page-411.htm

\section{Pour citer cet article :}

Frédérique Vernay, Jean-Yves Roussey« Présentation d’un support informatique de reconnaissance de mots écrits pour des enfants non verbaux avec autisme », Enfance 2012/4 (N4), p. 411-422. DOI $10.4074 / \mathrm{S} 0013754512004041$

Distribution électronique Cairn.info pour NecPlus.

(C) NecPlus. Tous droits réservés pour tous pays.

La reproduction ou représentation de cet article, notamment par photocopie, n'est autorisée que dans les limites des conditions générales d'utilisation du site ou, le cas échéant, des conditions générales de la licence souscrite par votre établissement. Toute autre reproduction ou représentation, en tout ou partie, sous quelque forme et de quelque manière que ce soit, est interdite sauf accord préalable et écrit de l'éditeur, en dehors des cas prévus par la législation en vigueur en France. Il est précisé que son stockage dans une base de données est également interdit. 


\title{
Présentation d'un support informatique de reconnaissance de mots écrits pour des enfants non verbaux avec autisme
}

\author{
Frédérique VERNAY et Jean-Yves ROUSSEY ${ }^{1}$
}

\begin{abstract}
RÉSUMÉ
Les difficultés que rencontrent les personnes avec autisme pour communiquer avec autrui sont souvent mises en relation avec un fonctionnement cognitif particulier fondé par exemple sur de meilleures capacités de traitement des stimuli visuels que des stimuli auditifs. Cet article présente un dispositif prenant en compte les capacités de traitement des enfants non verbaux avec autisme pour leur permettre de reconnaitre des mots écrits. Ce dispositif assisté par ordinateur, est basé sur la présentation de mots écrits en association avec leurs formes orales et leurs référents. Il mérite d'être testé systématiquement pour explorer les possibilités d'un accès aux symboles arbitraires chez les personnes non verbales avec autisme.
\end{abstract}

MOTS CLÉS : AUTISME, RECONNAISSANCE DE MOTS, LANGAGE ÉCRIT, DiSPOSITIF D’APPRENTISSAGE ASSISTÉ PAR ORDINATEUR

\begin{abstract}
Computer-aided recognition of written words by non verbal children with autism

The difficulties of persons with autism to establish a verbal or non verbal communication with others are often considered as related to specific cognitive performance, more specifically to a more efficient processing of visual stimuli as compared to auditory ones. This paper presents a protocol adapted to the type of processing of non verbal children with autism to allow them to recognize written words. In this protocol, written words are associated with their oral and pictorial counterpart via a computer-aided presentation. This protocol is intended to be tested in a systematic way so as to explore how far an access to written symbols is possible for non verbal persons with autism.

KEY-WORDS: AUTISM, WORDS RECOGNITION, WRITTEN LANGUAGE, SET-UP FOR COMPUTERAIDED LEARNING
\end{abstract}

${ }^{1}$ Psyclé (EA 3273) Aix-Marseille Université IUFM, 2 avenue Jules Isaac, 13626 Aix en Provence.Emails : f.vernay@aix-mrs.iufm.fr ; jy.roussey@aix-mrs.iufm.fr 


\section{INTRODUCTION}

La dénomination " trouble envahissant du développement » inscrit clairement l'autisme dans un processus développemental. Dans différentes classifications (CIM 10, 2006 ; DSMIV, 1994), il est caractérisé par l’altération évidente de la communication verbale. Plus précisément, les symptômes définissant ce trouble envahissant du développement sont regroupés dans la «triade de Wing 》 (Wing \& Gould, 1979) qui affecte les interactions sociales, la communication verbale et non verbale ainsi que les centres d'intérêt qui sont restreints et/ou les conduites fortement répétitives.

Pour ce qui est de la communication verbale, différentes capacités sont nécessaires à l'émergence du langage oral comme l'attention conjointe, la possibilité d'imitation (Nadel, 2011) ou le maintien de l'attention d'autrui (Bruner \& Haste, 1987). Or certaines de ces capacités semblent déficitaires chez l'enfant avec autisme (Baron-Cohen, 1995 ; Frith, 1992, 2003 ; Malvy, Adrien \& Sauvage, 1997).

Par ailleurs, la communication et les interactions sociales interviennent dans un contexte dont le traitement implique des compétences concernant notamment le partage de ses intentions et de ses émotions (Kasari, Sigman, Mundy \& Yirmiya, 1990). À ce niveau, les personnes avec autisme éprouvent à la fois des difficultés d'expression (Brun, Nadel \& Mattlinger, 1998), d'identification (Hobson, 1986a, 1986b) et de partage des émotions (Kasari et al., 1990). Selon Hobson (1993), ce déficit primaire de la réactivité socio-émotionnelle perturberait l'entrée en relation avec autrui.

De plus, le traitement du contexte dans les situations de communication requiert chez les interlocuteurs la perception et l'intégration simultanées de stimuli de nature variée (stimuli auditifs, visuels, kinesthésiques) et dont la pertinence est variable. Les enfants avec autisme auraient des difficultés à trier les informations pertinentes parmi toutes celles qui leur sont accessibles en raison d'une faiblesse en cohérence centrale, résultant d'un biais perceptif et qui les amèneraient à se cantonner à un traitement local des informations perçues (Frith, 1989) aux dépens d'un traitement global. Cependant, l'effet de ce biais diminue si les individus sont explicitement invités à traiter l'information de façon globale (Happé \& Frith, 2006). Pour leur part Mottron, Dawson, Soulières, Hubert et Burack (2006), dans leur proposition sur le surfonctionnement perceptif (Enhanced Perceptual Functioning), considèrent que la focalisation sur le traitement local reflète une supériorité générale des processus perceptifs de bas niveau. Cette proposition est étayée par les résultats de Plaisted, O’Riordan et Baron-Cohen (1998) qui ont montré que les personnes avec autisme sont davantage portées à traiter les traits spécifiques d'un stimulus que les traits partagés avec d'autres stimuli. Ce faisant, ils manifestent une meilleure discrimination associée à une difficulté à traiter les similitudes, à catégoriser l'information ou comme l'a constaté Mottron (2004) à hiérarchiser des stimuli. Ce constat est compatible avec les observations de Plumet, Hugues, Tardif et Mouren-Siméoni (1998), 
qui invoquent un déficit des fonctions exécutives pour rendre compte des difficultés à réaliser des tâches qui demandent des capacités d'anticipation ou de planification de situations nouvelles. Il faut noter que, malgré les déficits avérés dans les fonctions exécutives, le fonctionnement de la mémoire ne semble pas être globalement perturbé chez les individus avec autisme. Seuls certains déficits spécifiques sont signalés par différentes études. Gras-Vincendon, Bursztejn et Danion (2008) par exemple, ne constatent qu'un déficit limité de la mémoire à court terme et de la mémoire de travail dans des tâches spatiales et complexes chez les autistes qui de ce fait utilisent peu ou pas de stratégies d'organisation du matériel à mémoriser.

Ces difficultés de traitement des informations dans un contexte communicatif sont amplifiées par le caractère fugace de la communication orale qui implique un traitement en temps contraint des multiples informations (Gepner, 2006). Elles ont au moins deux conséquences. La première est une entrave à l'acquisition de la langue orale qui, à ses débuts, est habituellement acquise en situation d'interactions " naturelles ». La seconde est l'absence de maîtrise de l'écrit. En effet, le processus normal d'acquisition de la langue écrite en milieu scolaire prend largement appui sur les compétences orales (Alegria, Pignot, \& Morais, 1982). De nombreux travaux ont mis en évidence les liens entre les performances des enfants dans des tâches de conscience phonologique et leurs performances ultérieures en lecture (Bus \& van Ijzendoorn, 1999 ; Lambert \& Doyen, 2003 ; Sprenger-Charolles \& Casalis, 1996). Plus précisément, la maîtrise de la correspondance phonie-graphie est installée progressivement. En début d'apprentissage de l'écrit, quand l'enfant considère que l'écrit a pour fonction de coder du sens (Beau, 1994 ; Vallières, 1997), les mots sont traités à un niveau logographique ou visuo-sémantique sans lien avec la phonologie (Bastien, 1997). Ensuite, quand le mot est censé coder du son, l'enfant est confronté au problème de la segmentation pour déterminer ce qui dans le mot écrit, correspond à un segment prononçable (Bastien-Toniazzo, Magnan, \& Bouchafa, 1999). Il cherche alors à extraire de la chaîne graphique du mot des configurations correspondant aux syllabes orales parce qu'il peut les percevoir et les émettre (Bastien-Toniazzo, Magnan, \& Bouchafa, 1996).

Si les difficultés cognitives empêchant l'acquisition de la langue orale par les enfants avec autisme sont avérées, il n'en est pas de même pour ce qui est de l'apprentissage de l'écrit. Au contraire des travaux ont mis en évidence les bonnes capacités de traitement des informations visuelles des enfants avec autisme, qu'il s'agisse des traitements perceptifs (Peeters, 1996) ou des habiletés supérieures caractéristiques de l'hyperlexie (Mottron et al., 2006).

Dès lors il est possible de se demander si inverser le processus d'acquisition de l'articulation langue orale/langue écrite n'aboutirait pas à une meilleure maîtrise de l'écrit qui pourrait servir d'appui à l'apprentissage de l'oral. C'est le présupposé sous-jacent à cette recherche exploratoire qui a pour objectif de favoriser l'apprentissage et la reconnaissance de mots écrits par des enfants non verbaux avec autisme. Le moyen retenu pour tenter d'atteindre cet objectif réside 
dans l'élaboration d'un dispositif spécifique propre à réduire l'effet des troubles et des difficultés éprouvées par les enfants avec autisme dans des situations de communication orale ordinaires, et qui leur permette de prendre en compte, comme ils en sont capables, des liens dyadiques du type mots écrits et signifiants sonores (Planche, Lemonnier, Moalic, Labous, \& Lazartigues, 2002) ou des appariements perceptifs entre des éléments simples de type lettres et sons isolés (Mottron et al., 2006).

Ce dispositif utilise un support informatique. Le recours à ce type de support présente quatre avantages. Tout d'abord, cet outil permet de mettre en lien l'écrit et l'oral sous des formes stables (police, synthèse vocale) qui facilitent l'identification des signifiants oraux toujours présentés dans le même contexte et sous la même forme phonique contrairement aux conditions habituelles de perception de la langue orale. Un autre avantage réside dans la possibilité d'adapter la présentation de ces formes systématisées (notamment la durée, la vitesse de présentation, la taille des caractères) aux capacités connues des enfants avec autisme. Un troisième avantage de l'outil informatique est lié à la présentation du matériel écrit et oral dans un contexte épuré qui devrait permettre aux participants de profiter de leurs habiletés perceptives pour associer les seuls stimuli visuels et sonores utiles à la tâche et présentés successivement. En effet, cette association peut être réalisée à partir du traitement séquentiel d'un nombre de stimuli inférieur au seuil de trois repéré par Planche et al. (2002) chez les enfants avec autisme. Enfin, un quatrième avantage, compte tenu des difficultés à identifier les émotions (Hobson, 1986a, 1986b), ainsi qu’à les partager (Kasari et al., 1990) réside dans la faible charge émotionnelle de cet outil comparativement à celle représentée par un interlocuteur « humain ». En effet, la présentation des mots à reconnaître en passation individuelle sur un support numérique permet de proposer des situations demandant moins de mobilisation de compétences socio-émotionnelles que des situations ordinaires d'interactions enseignant/élève notamment dans la gestion des réussites et des échecs du participant dans les tâches qui sont directement gérés par le programme informatique.

Au total, l'outil informatique devrait permettre une présentation multimodale systématique et répétitive, dans un contexte épuré, d'une photo, d'un mot écrit et de sa forme sonore correspondante, ce qui devrait faciliter la mise en lien des stimuli présentés et donc favoriser l'accès à leur signification commune.

\section{DESCRIPTION DU DISPOSITIF}

Le dispositif requiert un ordinateur avec écran tactile sur lequel les participants travaillent individuellement (figure 1). Ils agissent directement sur l'écran tactile pour répondre aux consignes et n’ont pas à apprendre à manipuler la souris de l'ordinateur.

Un programme spécialement conçu pour l'expérimentation permet, grâce au recours à une synthèse vocale, de contrôler la prosodie de la parole et d'éviter les variations inter ou intra individuelles, favorisant ainsi la perception du message 


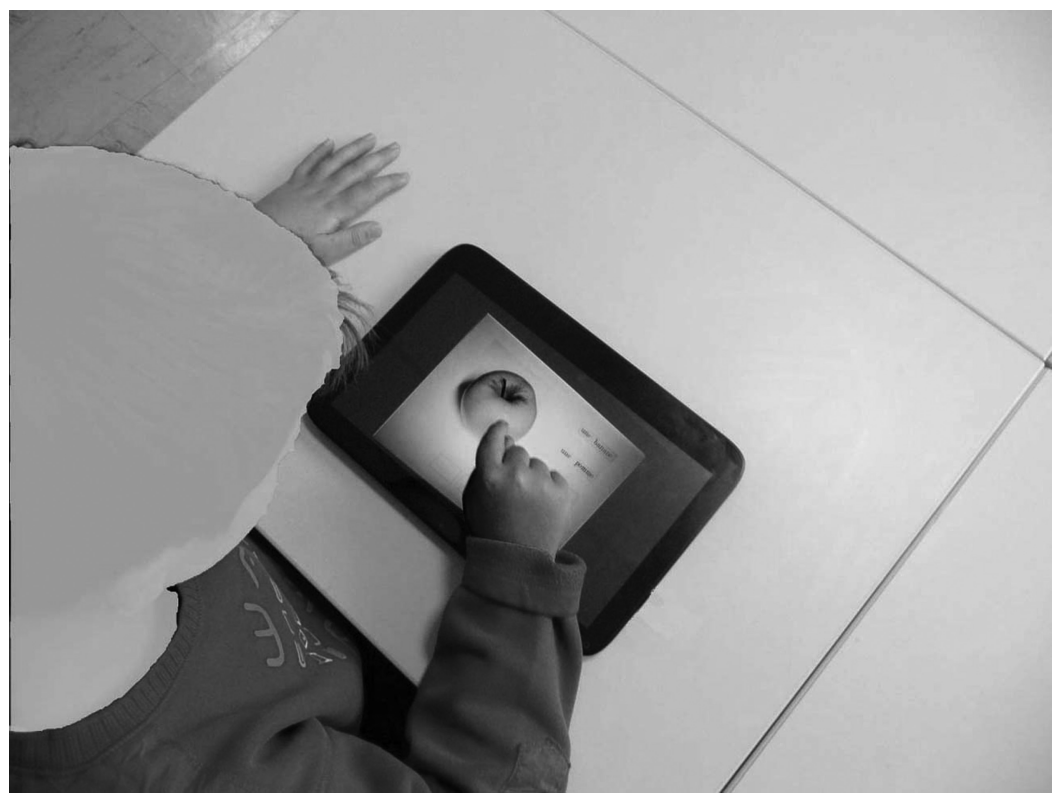

\section{Figure 1.}

Participant utilisant le dispositif

oral par les participants. En effet, la même voix est enregistrée pour tous les mots, et chaque mot écrit a ainsi un correspondant sonore stable.

Ce programme permet également une association systématique mot écrit/mot oral/référent (photo). Cette association systématique des mots écrits ou entendus avec leur référent facilite la mise en relation entre le stimulus écrit, le signifiant oral et le signifié qui est censée favoriser l'accès au sens et la reconnaissance des mots proposés.

\section{Tâche expérimentale}

Trois tâches de reconnaissance de mots écrits peuvent être proposées aux élèves avec ce dispositif. Elles sont de difficulté variable selon que les participants doivent retrouver le stimulus parmi une partie ou la totalité des mots utilisés et selon que pour ce faire, ils disposent ou non de l'affichage à l'écran du mot stimulus. Concrètement dans la tâche la plus facile les participants doivent retrouver le stimulus parmi trois mots cibles et peuvent se référer au stimulus présent à l'écran pendant toute la recherche. Dans la tâche de difficulté intermédiaire ils doivent retrouver le stimulus parmi l'ensemble du matériel verbal, tout en disposant de la présence à l'écran du modèle. Dans ce cas, l'accroissement de la difficulté résulte de cette augmentation de l'espace de recherche. Dans la tâche la plus difficile, la recherche est faite parmi l'ensemble des mots, le stimulus n'étant plus présent à l'écran. C'est là l'absence de modèle qui accroît encore la difficulté de la tâche. En effet, elle impose le recours à une 
représentation mentale du stimulus stockée en mémoire de travail pour guider la reconnaissance qui requiert alors plus de ressources cognitives.

\section{Consigne}

La consigne, identique pour les trois tâches, demande au participant dans un premier temps de «pointer sur l'écran le mot écrit ou la photo pour entendre le mot», puis d'« appuyer sur la flèche pour continuer» ensuite d' "appuyer sur le mot que tu viens de voir et d'entendre » et enfin « d'appuyer sur la flèche pour passer à un nouveau mot».

Un entraînement permet de s'assurer que les élèves comprennent cette consigne et parviennent à réaliser la tâche. En cas de difficultés de compréhension au cours de cet entraînement, l'expérimentateur exécute la tâche en modèle avant de la resoumettre au participant. Compte tenu des résultats avancés par Nadel et al. (2011), ce modèle devrait permettre aux participants de réaliser la tâche. En effet, ces chercheurs montrent que des enfants avec autisme non verbaux d'un âge développemental de 36 mois peuvent mémoriser une séquence d'actions observées et la reproduire en différé totalement ou partiellement sur la base des représentations motrices engrangées sans expérience motrice simultanée à l'observation. Toutefois, si la difficulté à réaliser la tâche persiste, l'expérimentateur peut guider la main du participant en début d'entraînement.

\section{Matériel verbal}

Les mots proposés aux participants sont des mots concrets directement et aisément associables à des objets du monde réel (Tautelle, 1984) et choisis en vue de leur réutilisation ultérieure dans des phrases du type sujet/verbe/complément. Plus précisément, ces mots, au nombre de sept, sont trois prénoms, ceux des deux participants et d'un autre élève de la classe qui n'a pas participé à l'expérience et les quatre mots suivants : une, pomme, banane, mange. Ils sont présentés isolément (Akim, Eddy, Erwann, mange) ou en groupe (une banane, une pomme). En effet, Ferreiro et Teberosky (1982) qui ont repéré six étapes dans l'évolution du concept d'écriture chez l'enfant, ont montré que ce n'est qu'à la dernière étape que l'enfant considère que tous les mots écrits sont prononcés, notamment les articles qui auparavant sont intégrés aux substantifs qu'ils déterminent. Pour cette raison, mais aussi parce que les enfants avec autisme ont des difficultés à identifier la valeur syntaxique des mots entendus et tendent à produire des énoncés agrammatiques, avec un choix arbitraire des éléments pertinents du message (Mottron 2004), les substantifs sont présentés avec leurs articles.

L'ordre de présentation de ces mots, variable en fonction des tâches, est fixé aléatoirement à chaque passation.

Par ailleurs, d'autres mots présentant les mêmes caractéristiques (trois prénoms : Line, Louisa, Mael et trois mots concrets : une poire, une tomate, couper) sont utilisés pour entraîner les participants afin de vérifier qu'ils comprennent bien les trois tâches et la consigne. 
Pour chaque mot ou groupe de mots à identifier, la présentation est réalisée à l'aide de trois diapositives.

La première diapositive comprend dans le quart inférieur gauche le mot ou le groupe de mots écrit en Arial 40 et dans le quart supérieur gauche la photographie $(13,3 \mathrm{~cm}$ X 11,5 cm). Un lien peut être activé par le pointage de la photographie ou du mot écrit qui déclenche l'émission de la forme sonore du stimulus par la synthèse vocale. Dans le coin inférieur droit un pictogramme en forme de flèche déclenche le passage à la diapositive suivante lorsqu'il est pointé.

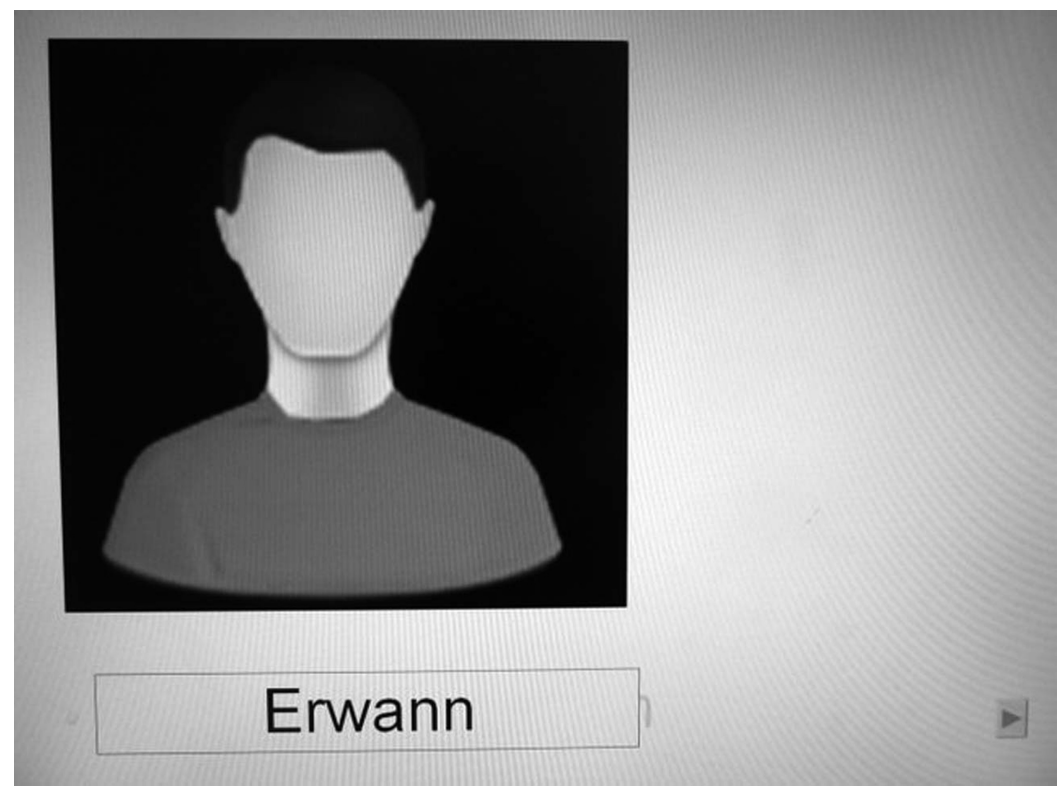

Figure 2.

Écran de présentation du stimulus et de son référent

La deuxième diapositive varie selon le niveau de difficulté de la tâche. Au niveau le plus facile, la moitié gauche de la diapositive est identique à celle de la première et la moitié droite contient soit les 3 prénoms, soit les 3 autres mots ou groupes de mots présentés verticalement selon un ordre fixé aléatoirement et écrits en Arial 18, parmi lesquels le participant doit reconnaître et pointer le stimulus.

Au niveau suivant, la moitié gauche de la diapositive reste identique et la moitié droite contient l'ensemble des mots utilisés.

Enfin, au niveau le plus difficile, le quart inférieur gauche de la diapositive ne contient que l'emplacement vide du mot stimulus tandis que la photographie est toujours affichée dans le quart supérieur gauche et l'ensemble des mots utilisés présents dans la moitié droite.

La troisième diapositive, elle aussi, diffère selon le niveau de difficulté. Pour les deux premiers niveaux sa moitié gauche contient en haut la photographie et 


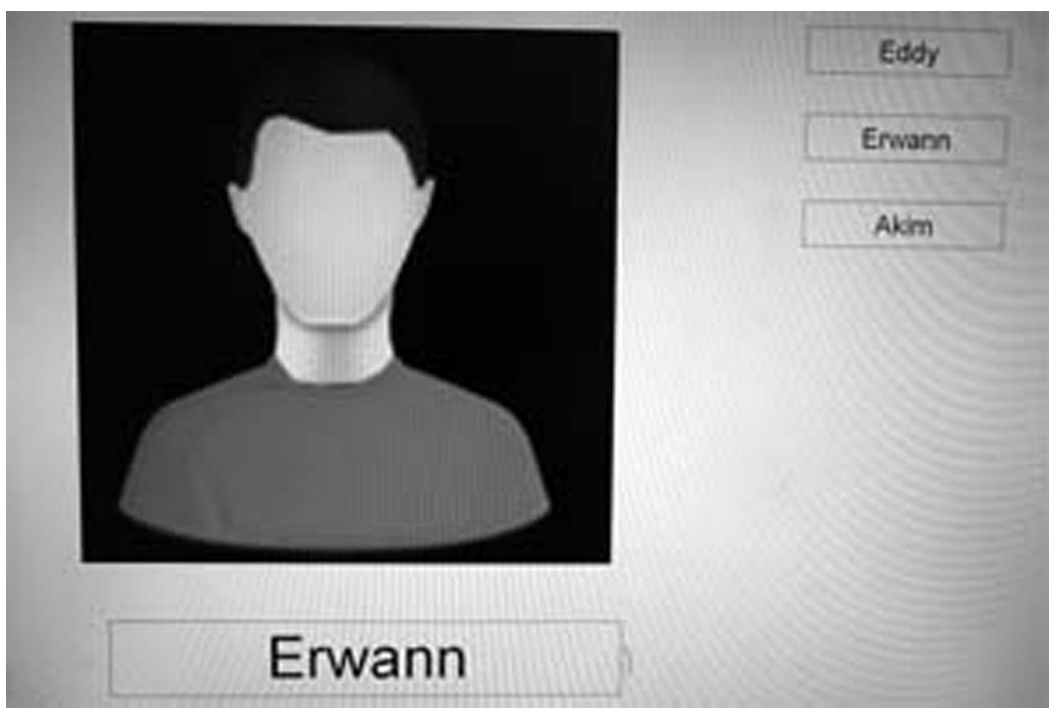

Figure 3.

Écrans lors de la recherche de la cible du plus simple au plus complexe

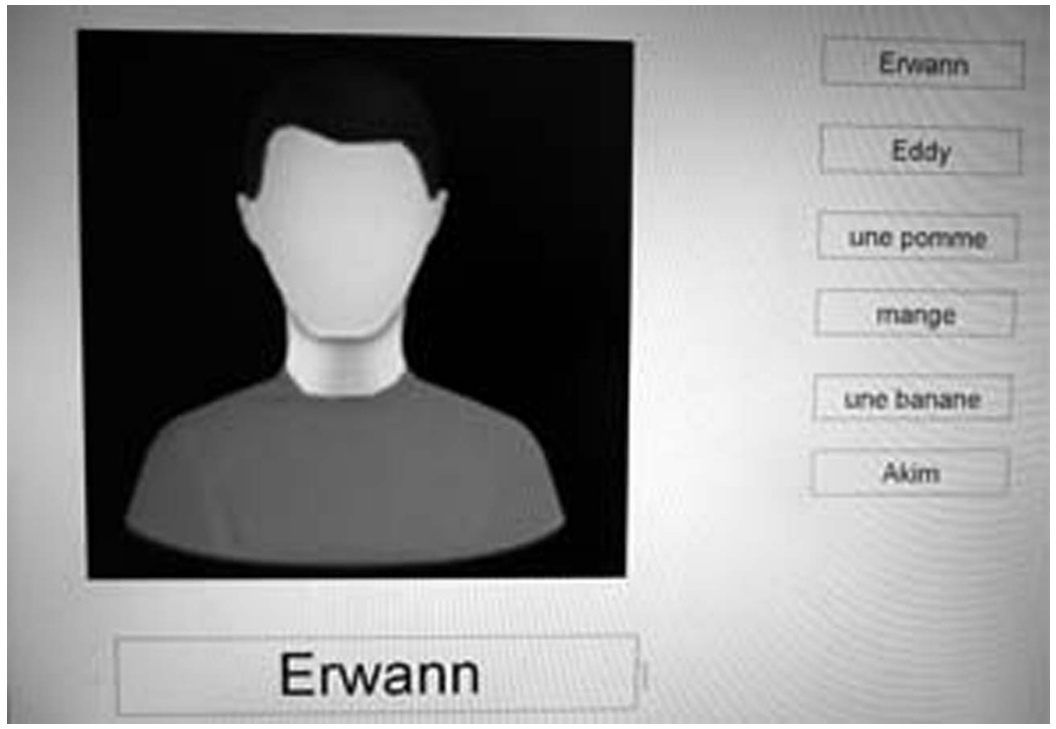

\section{Figure 4.}

Écrans lors de la recherche de la cible du plus simple au plus complexe

en bas affiché dans son emplacement le stimulus en caractères gras tandis que le mot cible lui aussi écrit en gras reste seul dans la moitié droite.

Dès l'affichage de cette diapositive, la synthèse vocale émet la forme sonore du mot écrit. Pour le niveau le plus difficile, à l'affichage, la moitié gauche de 


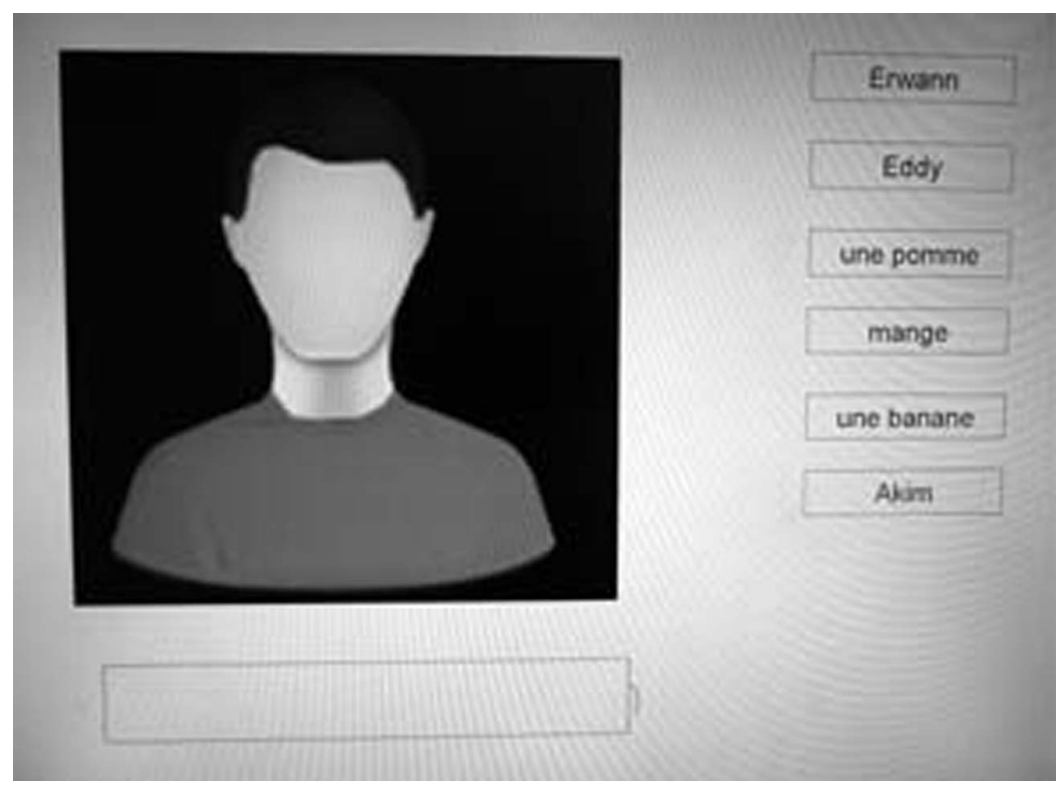

Figure 5.

Écrans lors de la recherche de la cible du plus simple au plus complexe

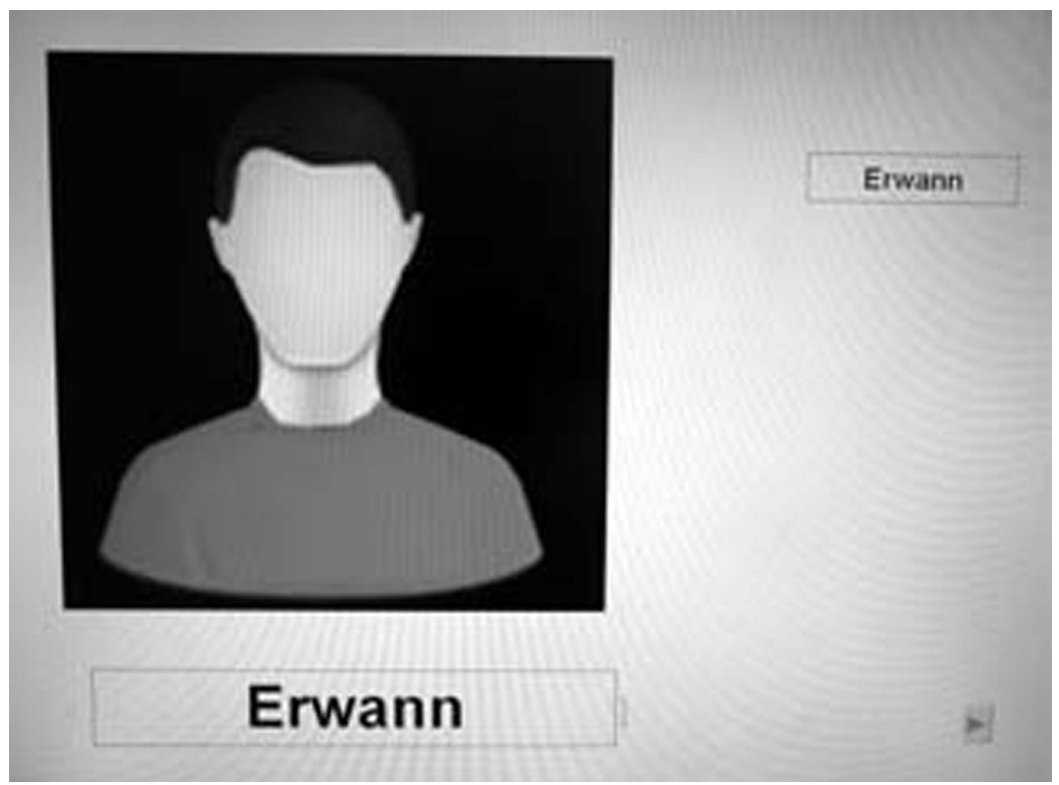

Figure 6.

Écrans lors de la recherche de la cible du plus simple au plus complexe 


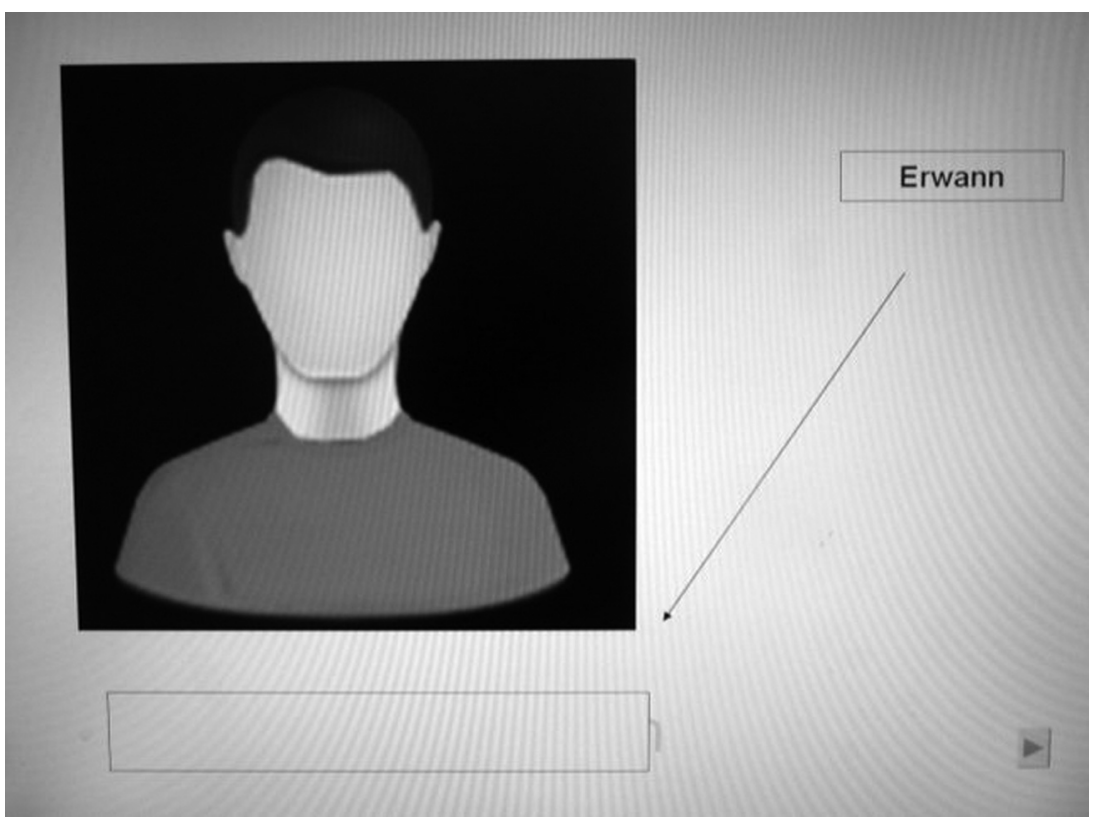

Figure 7.

la diapositive contient en haut la photographie et en bas l'emplacement vide du stimulus tandis que le mot cible est affiché en caractères gras dans la moitié droite avant de se déplacer jusqu'à l'emplacement vide du quart inférieur gauche.

Dès que ce déplacement est achevé, la synthèse vocale émet la forme sonore du mot écrit. Pour les trois tâches, le pictogramme déjà utilisé sur la première diapositive est présent dans le coin inférieur droit.

Le passage entre deux diapositives d'une même séquence de trois ou entre la dernière et la première diapositive de deux séquences successives est déclenché par le pointage du pictogramme en forme de flèche quand il est présent. Dans le cas contraire (passage entre les deuxième et troisième diapositives d'une série), le passage vers la suivante est déclenché automatiquement par le pointage de la réponse quand cette dernière est bonne. En cas d'erreur c'est la première diapositive de la séquence qui est à nouveau affichée.

\section{Conclusion}

Les expérimentations projetées devraient pouvoir déterminer si le dispositif permet à des participants non verbaux avec autisme d'apprendre des mots écrits inconnus qu'ils pourront ensuite toujours reconnaître dans n'importe quel contexte ou simplement d'améliorer momentanément une compétence d'identification à l'œuvre dans cet environnement spécifique. Cette question est importante car elle pose celle de l'acquisition de la langue écrite et celle de l'accès à la langue orale grâce à la médiation de l'écrit pour les enfants avec autisme qui ne peuvent profiter des pratiques instituées d'enseignement de la langue. 


\section{Remerciements}

Remerciements au service de la recherche et du développement de l'IUFM d'Aix en Provence de l'Université de Provence qui a permis de financer cette recherche.

\section{RÉFÉRENCES}

Alegria, J., Pignot, E., \& Morais, J. (1982). Phonetic analysis of speech and memory codes in beginning readers. Memory and Cognition, 10, 451-456.

American Psychiatric Association (1994). DSMIV. Manuel diagnostique et statistique des troubles mentaux. Paris : Masson.

Baron-Cohen, S. (1995). Mindblindness, an essay on autism and theory of mind. Cambridge, Ma: MIT Press.

Bastien, C. (1997). Les connaissances de l'enfant à l'adulte. Paris : Armand Colin.

Bastien-Toniazzo, M., Magnan, A., \& Bouchafa, H. (1996). Une étude longitudinale des stratégies d'apprentissage de la correspondance grapho-phonologique en français. Revue de psychologie de l'éducation, 2, 37-65.

Bastien-Toniazzo, M., Magnan, A., \& Bouchafa, H. (1999). Nature des représentations du langage écrit aux débuts de l'apprentissage de la lecture : un modèle interprétatif. Journal international de psychologie, 34(1), 43-58.

Beau, A.-S. (1994). Identification du mot écrit chę l'adulte analphabète (mémoire de maîtrise non publié). Université de Provence, Aix-en-Provence, France.

Brun, P., Nadel, J., \& Mattlinger, M.-J. (1998). L'hypothèse émotionnelle dans l'autisme. Psychologie française, 2(43), 147-156.

Bruner, J., \& Haste, H. (1987). Making sense: The child's construction of the world. New York, NJ : Methuen.

Bus, A. G., \& van Ijzendoorn, M. H. (1999). Phonological awareness and early reading: A metaanalysis of experimental training studies. Journal of Educational Psychology, 91, 403-414.

Classification internationale des maladies et des problèmes de santé connexes (2006, dixième révision). Genève : OMS.

Ferreiro, E., \& Teberosky, A. (1982). Literacy before schooling. Exeter, NH: Heinemann.

Frith, U. (1992). L'énigme de l'autisme. Paris : Odile Jacob.

Frith, U. (2003). Autism. Explaining the enigma. Oxford: Blackwell publishing. (2 ${ }^{\text {nd }}$ ed., 1989).

Gepner, B. (2006). Le monde va trop vite pour les personnes autistes! Hypothèses neurophysiopsychopathogéniques et implications rééducatives. Neuropsychiatrie de l'enfance, 54(6-7), 371-374.

Gras-Vincendon, A., Bursztejn, C., \& Danion, J.-M. (2008). Fonctionnement de la mémoire chez les sujets avec autisme. L'Encéphale, 34(6), 550-556.

Happé, F., \& Frith, U. (2006). The weak central coherence account: Detail focused cognitive style in autism spectrum disorders. Journal of Autism and Developmental Disorders, 36, 5-25.

Hobson, R. P. (1986a). The autistic child's appraisal of expressions of emotion. Journal of Child Psychology and Psychiatry, 27(3), 321-342. 
Hobson, R. P. (1986b). The autistic child's appraisal of expressions of emotion: A further study. Journal of Child Psychology and Psychiatry, 27(5), 671-680.

Hobson, R. P. (1993). Understanding persons: The role of affects. In S. Baron-Cohen, H. Tager-Flusberg, \& D. J. Cohen (Eds.), Understanding other minds: Perspectives from autism (pp. 204-227). Oxford: Oxford University Press.

Kasari, C., Sigman, M., Mundy, P., \& Yirmiya, N. (1990). Affective sharing in the context of joint attention interactions of normal, autistic, and mentally retarded children. Journal of Autism and Developmental Disorders, 20(1), 87-100.

Lambert, E., \& Doyen, A.-L. (2003). Compétences phonologiques en maternelle et acquisition du langage écrit. In A. Vom Hofe, H. Charvin, J.-L. Bernaud, \& D. Guédon, (Eds.), Psychologie différentielle : recherches et réflexions (pp. 143-147). Rennes : Presses Universitaires de Rennes.

Malvy, J., Adrien, J.-L., \& Sauvage, D. (1997). Signes précoces de l'autisme et films familiaux. La psychiatrie de l'enfant, 40(1), 175-198.

Mottron, L. (2004). L'autisme, une autre intelligence. Diagnostic, cognition et support des personnes autistes sans déficience intellectuelle. Paris : Mardaga.

Mottron, L., Dawson, M., Soulières, I., Hubert, B., \& Burack, J. (2006). Enhanced perceptual functioning in autism : An update, and eight principles of autistic perception. Journal of Autism and Developmental Disorders, 36, 27-43.

Nadel, J., Aouka, N., Coulon, N., Gras-Vincendon, A., Canet, P., Fagard, J., \& Bursztejn, C. (2011). Yes they can! An approach of observational learning in low-functioning children with autism. Autism, 15(2), 421-435.

Nadel, J. (2011). Imiter pour grandir. Paris : Dunod.

Peeters, T. (1996). L'autisme: De la comprehension à l'intervention. Paris : Dunod

Plaisted, K., O’Riordan, M., \& Baron-Cohen, S. (1998). Enhanced visual search for a conjunctive target in autism: A research note. Journal of Child Psychology and Psychiatry, 39, 777-783.

Planche, P., Lemonnier, E., Moalic, K., Labous, C., \& Lazartigues, A. (2002). Les modalités de traitement de l'information chez des enfants autistes. Annales médicopsychologiques, 160, 559-564.

Plumet, M. H., Hugues, C., Tardif, C., \& Mouren-Siméoni, M.-C. (1998). L’hypothèse d'un déficit des fonctions exécutives dans l'autisme. Psychologie française, 43(2), 111-195.

Sprenger-Charolles, L., \& Casalis, S. (1996). Lire: lecture et écriture acquisition et troubles du développement. Paris : Presses Universitaires de France.

Tautelle, D. (1984). L'enfant à la rencontre du langage. Bruxelles : Mardaga.

Vallières, N. (1997). L'apprentissage de la lecture par des adultes analphabètes (mémoire de maîtrise non publié). Université de Provence, Aix-en-Provence, France.

Wing, L., \& Gould, J. (1979). Severe Impairments of Social Interaction and Associated Abnormalities in Children: Epidemiology and Classification. Journal of Autism and Developmental Disorders, 9(1), 11-29. 